\title{
Commissioning of the ATLAS Inner Detector with cosmic rays
}

\author{
E. Klinkby on behalf of the ATLAS collaboration \\ Niels Bohr Institute, HEP
}

\begin{abstract}
The tracking of the ATLAS experiment is performed by the Inner Detector which has recently been installed in its final position. ${ }^{1}$ Various parts of the detector have been commissioned using cosmic rays both on the surface and in the ATLAS pit. The different calibration, alignment and monitoring methods have been tested as well as the handling of the conditions data. Both real and simulated cosmic events are reconstructed using the full ATLAS software chain, with only minor modifications to account for the lack of timing of cosmics events, the lack of magnetic field and to remove any vertex requirements in the track fitters. Results so far show that the Inner Detector performs within expectations with respect to noise, hit efficiency and track resolution.
\end{abstract}

Keywords: Cosmics, ATLAS, Inner Detector, Commissioning

\section{Introduction}

Doing next year, the Large Hadron Collidor at CERN goes into operation providing proton-proton collisions at $14 \mathrm{TeV}$ in the center-of-mass system. Bunches are collided every $25 \mathrm{~ns}$ with an expected average number of collisions $\sim 25$ per bunch crossing when operating at the design luminosity. The high rate and energy set strong requirements for the tracking. In ATLAS the requirements are met by the Inner Detector operating in a $2 \mathrm{~T}$ magnetic field. The Inner Detector has a typical layered structure consisting of three sub-detectors based on different detector technologies to best cope with the requirements. Each sub-detector consists of a barrel part and two end-caps - the measurement accuracies quoted below corresponds to those of the barrel detectors from which most of the results presented below originates.

Closest to the interaction point is the very radiation hard and fine segmented pixel detector (Pixel) providing three measurements for each track of $12 \mu \mathrm{m}$ precision in $r \phi$ and $110 \mu \mathrm{m}$ in the $(r z)$ plane. The next sub-detector met by a traversing particle consists of four silicon layers of the SemiConductor Tracker (SCT) - a silicon micro-strip detector with a readout pitch of $80 \mu \mathrm{m}$, which yields a binary resolution on the detector of $\sim 21 \mu \mathrm{m}$. 
The cylindrical volume extending from a radius of $56 \mathrm{~cm}$ to $107 \mathrm{~cm}$ is covered by the gaseous Transition Radiation Tracker (TRT). Based on the principle of transition radiation the TRT consists of 73 layers of straws interleaved with radiator material providing both continuous tracking as well as electron identification by their transition radiation signature. The intrinsic measurement accuracy is expected to be $170 \mu \mathrm{m}$ in the $(r \phi)$ plane. Presently the Inner Detector is installed in the pit and commissioning is ongoing. Results from standalone sub-detector measurements at the surface as well as combined tests in the ATLAS cavern are presented below.
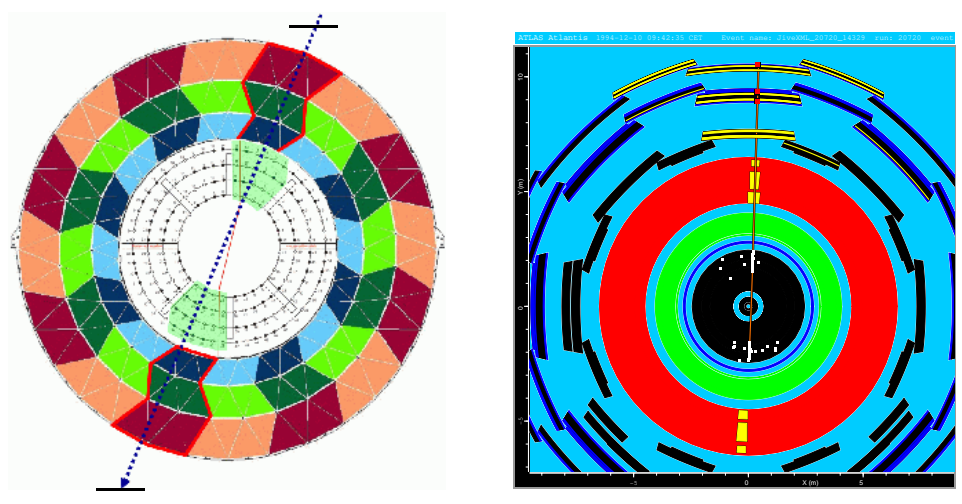

Fig. 1. Left: Setup for cosmic data taking at the surface. Scintillator plates are placed above and below the detector. Only the shaded sectors are read out. Right: Event display showing an event from cosmics in the pit. Note how the track is formed based on the information of many sub-detectors.

\section{Commissioning using Cosmic rays: At the surface}

For the most part, cosmic rays at the surface consist of low energy muons (average $2 \mathrm{GeV}$ ) produced in the atmosphere. Since the arrival time of such muons is random, the surface setup for the integrated TRT cosmics included scintillator plates used for triggering. Plates were placed above and below the sub-detectors (see figure 1) in such a way that a coincidence would require a particle passing near the center of the detector and therefore to some extent, resemble a particle produced in a beam collision - at least with respect to which detector parts are passed. Offline, the full event reconstruction is performed on real cosmic data as well as simulations of the same using as much as possible standard ATLAS software packages. Minor updates were needed to cope with the fact that the cosmic events are 
not synchronized with the ATLAS clock, and for the tracking to perform without vertex constraints or magnetic field. The resulting hit efficiencies for the TRT- and SCT barrel tests were within expectations - see figure 2. Additional tests using a random triggers to record noise levels were also successful - results for the SCT (barrel and endcap) and the Pixel (endcap) are shown in figure 3 . After alignment and calibration the resolution of the TRT and SCT tracks are $\sigma=59 \mu \mathrm{m}$ and $\sigma=170 \mu \mathrm{m}$ respectively which is within expectations (see figure 4). The reason that SCT the uncertainty is larger than the ATLAS expectations is partly due to the low momenta of the cosmic rays, and partly that no magnetic field was applied, and therefore the particle momentum could not be measured and effects of multiple interactions could not be corrected for. As for the TRT, the fact that the expectations for the final ATLAS setup are reached at the cosmics tests indicate that improvements with respect to the design value can be expected in the final setup.
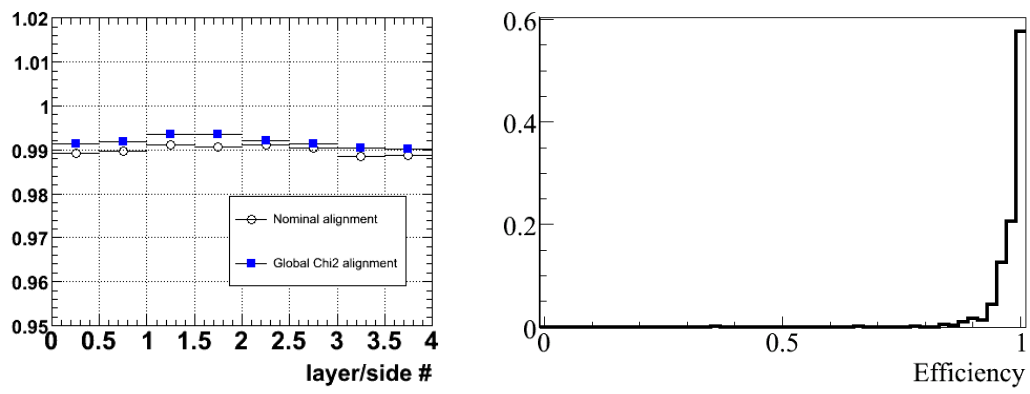

Fig. 2. Hit efficiencies of the SCT(left) and TRT(right) as measured in tests on the surface.

\section{Commissioning using Cosmic rays: In the pit}

Recently the Inner Detector has been installed in the ATLAS pit and tests performed with parts of the SCT and TRT integrated into the ATLAS data acquisition. Figure 5(a) shows an event from the test run. The muon chambers and tile calorimeter are used as triggers and this has caused some difficulties: This usage of the tile calorimeter was not part of the design requirements and the timing of the different muon modules has not been made uniform yet. However these issues presently being addressed. Simultaneously a different approach is being prepared for the next runs, where scintillator plates will be placed on top of the ATLAS detector. For practical reasons however the area covered with scintillators will be small, 

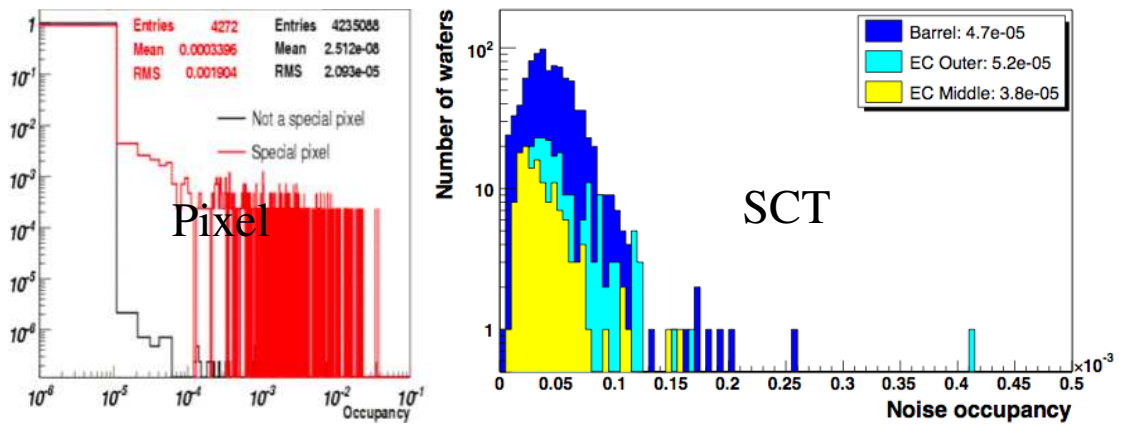

Fig. 3. Noise levels of the Pixel and SCT sub-detectors as measured in tests on the surface. The latter was tested only under "warm" conditions, where the noise occupancy is expected to be larger than during ATLAS operation.
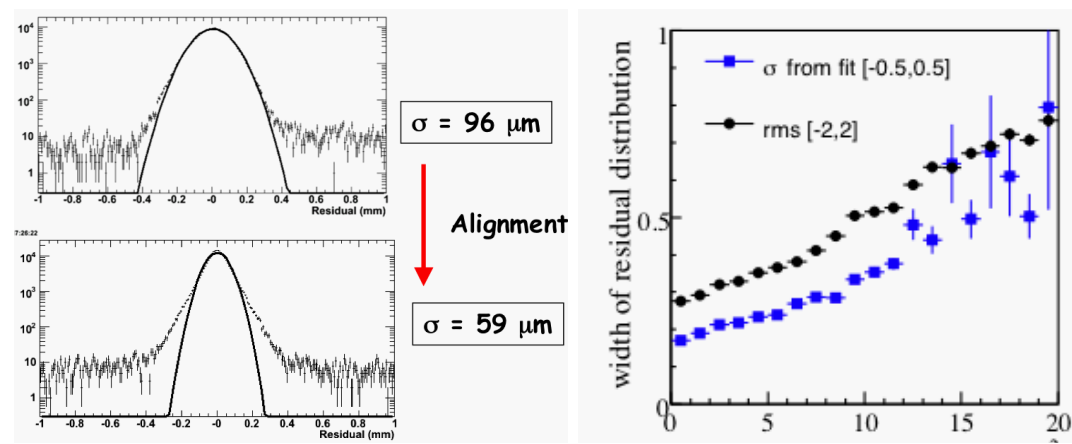

Fig. 4. Left: SCT track residuals before and after alignment. Right: $\sigma$ of the TRT track residual distribution as a function of the unbiased $\chi^{2}$ for each given hit (i.e. the $\chi^{2}$ of the track removing the contribution of the hit under evaluation). Both plots stem from tests performed at the surface.

causing a low event rate from this cosmic trigger. The results of the most recent cosmic run in terms of TRT noise and resolution are shown in figure 5. 

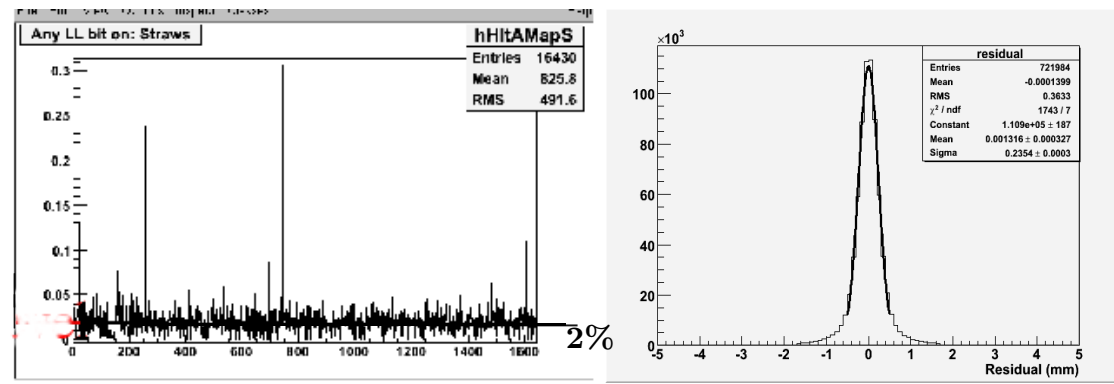

Fig. 5. Left: Noise levels of the various straws in one TRT $\phi$ sector. Level is generally below the expected 3\%. Right: TRT track residuals as measured in the ATLAS cavern. The resolution is worse than the specifications $(170 \mu \mathrm{m})$ due to the fact that multiple scattering is unaccounted for.

\section{Conclusions}

The ATLAS Inner Detector is being commissioned with cosmic rays. The full data acquisition chain is being used and cosmics data as well as simulation is reconstructed with standard ATLAS software. The results in terms of noise, hit efficiency and track resolution fall well within specifications. However the integration of the remaining parts of the Inner Detector into the ATLAS data acquisition is ongoing and the detector commissioning will proceed in to coming months.

\section{References}

1. ATLAS: Detector and physics performance technical design report. Volume 1 CERN-LHCC-99-14 